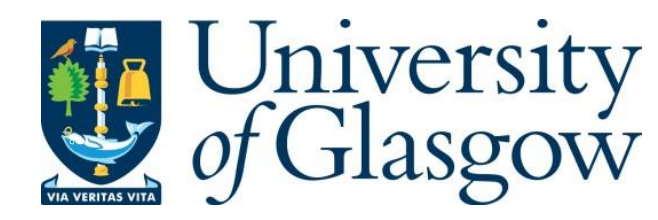

Tyboroski, M., Macêdo, R. and Camley, R. E. (2021) Nonreciprocity in millimeter wave devices using a magnetic grating metamaterial. Physical Review Materials, 5(11), 115201.

There may be differences between this version and the published version. You are advised to consult the publisher's version if you wish to cite from it.

http://eprints.gla.ac.uk/258446/

Deposited on: 5 November 2021

Enlighten - Research publications by members of the University of Glasgow http://eprints.gla.ac.uk 


\title{
Nonreciprocity in Millimeter Wave Devices Using a Magnetic Grating Metamaterial
}

\author{
Markus Tyboroski ${ }^{1}$, Rair Macêdo ${ }^{2,1 *}$, and Robert E. Camley ${ }^{1}$ \\ ${ }^{1}$ Center for Magnetism and Magnetic Materials, Department of Physics and Energy Science, \\ University of Colorado at Colorado Springs, Colorado Springs, Colorado 80918, USA \\ ${ }^{2}$ James Watt School of Engineering, Electronics \& Nanoscale Engineering Division, University of \\ Glasgow, Glasgow G12 8QQ, United Kingdom \\ *Rair.Macedo@glasgow.ac.uk
}

\begin{abstract}
The control and manipulation of many of light's fundamental properties, such as reflectivity, has become a topic of increasing interest since the advent of engineered electromagnetic structures - now known as metamaterials. Many of these metamaterial structures are based on the properties of dielectric materials. Magnetic materials on the other hand, have long been known to interact with electromagnetic waves in unusual ways; in particular, their nonreciprocal properties have enabled rapid advances in millimeter wave technology. Here, we show how a structured magnetic grating can be employed to engineer electromagnetic response at frequencies upwards of hundreds of GHz. In particular, we investigate how nonreciprocal reflection can be induced and controlled in this spectral region through the composition of the magnetic grating. Moreover, we find that both surface and guided polaritons contribute to high-frequency nonreciprocity; the nature of these is also investigated. Control of electromagnetic radiation at high frequencies is a current challenge of communications technology, where our magnetic gradient might be employed in devices including signal processing filters and unidirectional isolators.
\end{abstract}

KEYWORDS: millimitre wave, RF devices, grating metamaterial, ATR, nonreciprocal reflection 


\section{INTRODUCTION}

Magnetic materials have long been used for a variety of microwave applications including circulators and isolators for radar systems and mobile telephone relay stations [1,2]. Some of the main properties of magnets that enable such versatility are their negative effective permeability [3-5] and non-reciprocal response [3,6] near their ferromagnetic resonance frequency. This means that their electromagnetic characteristics depend on the direction of motion of the wave crossing through them. For just as long, ferrites and garnets - in particular, yttrium iron garnet (YIG) - have been the firstchoice materials for most of these applications $[7,8]$. The interest in this class of materials stems from their typical operating frequency in the low GHz frequency range, their low damping. as well as their non-metallic behavior.

With the advent of new information technologies, such as $5 \mathrm{G}$ and even $6 \mathrm{G}$, high frequency bands, from the high $\mathrm{GHz}$ to the $\mathrm{THz}$ range, are now being considered in order to enable data rates in the order of hundreds of Gbps [9]. Thus, making it now necessary to develop new high-frequency operating devices [10] including on-chip THz isolators [11], amplifiers and oscillators [12]. This brings about three main drawbacks when incorporating magnetic materials into electronic devices:

1) The aforementioned class of materials is no longer the obvious choice as they would require extremely large applied magnetic fields in order to obtain such high operating frequencies $[6,13,14]$;

2) Antiferromagnetic materials, which can have sub-terahertz operating frequencies, typically require extremely low temperature [15]; and

3) Many of the other candidates are metallic structures wherein the electron motion induced by the electromagnetic waves near the surface of the magnet prevents the interior of the sample from interacting with the wave [16].

To circumvent these problems, various techniques have been employed or investigated. These include using materials with high anisotropy such as barium doped hexagonal ferrites [17-19] or using thin metallic films where eddy current damping is minimized [14, 20]. 
More recently, the emergence of artificial materials designed for novel electromagnetic wave propagation, known as metamaterials [21], has enable completely new solutions to this problem. The term 'metamaterial' was initially used to describe electromagnetic structures that possess both negative permittivity and negative permeability [22]—also known as left-handed metamaterials [21]. However, it has now become a synonym of artificial structures engineered to have specific physical properties in just about any field [23]. For instance, different metamaterial designs have been recently used to make advances in topics such as thermal-controlled chirality [24] and near-perfect light absorption [25]. In magnetism, for instance, it was recently shown that constructing metamaterials comprising canted antiferromagnetically aligned layers [26] could enable novel high-frequency microwave devices. In addition, by using ultra-thin films which have operating frequencies [27] above $50 \mathrm{GHz}$ due to exchange spin-wave resonances [28]. Nonetheless, these newer classes of materials either have frequencies typically below $50 \mathrm{GHz}$, require significant precision in manufacturing, or have nonreciprocal effects which are relatively small.

In contrast, in this paper we show that by structuring thicker magnetic metallic films into a magnetic grating, it is possible to considerably reduce the conductivity of the overall structure as well as to engineer strong nonreciprocal responses across a wide range of frequencies. A particularly exciting feature that we demonstrate is nonreciprocal behavior at frequencies well above $300 \mathrm{GHz}$ for these structured metallic systems. This is quite surprising since metallic ferromagnets typically have resonances in the $10-25 \mathrm{GHz}$ range.

We demonstrate our findings through attenuated total reflection (ATR) calculations. We show that the frequency regions of high absorption and significant nonreciprocity can be substantially modified by changing the filling fraction of grating. To understand the reflectivity results, we calculate the dispersion relation of magnetic polariton modes of an insulator/structured metal/insulator structure and connect their spatial profiles to the nonreciprocal reflectivity spectra. 


\section{THEORETICAL CALCULATIONS}

We will soon discuss results for reflectivity (with a particular focus on ATR) as well as nonreciprocity of magnon modes, but before discussing our main findings, we will discuss the structure of interest as well as the main aspects of the electromagnetic theory through which we model reflectivity.

\subsection{The Magnetic grating}

Here, we will investigate the case of a structured magnetic metallic film. The structuring takes form of a supercell of a binary-grating consisting of iron $(\mathrm{Fe})$ nano-bars separated by a dielectric, as shown in Figure 1(a). This structuring method has been employed to construct a variety of metamaterials; this method has been more prominently in the field of metasurfaces where either plasmonic or phononic structures have been engineered to control reflection [29], transmission [30], and the phase profiles of electromagnetic waves [31] at various frequencies.
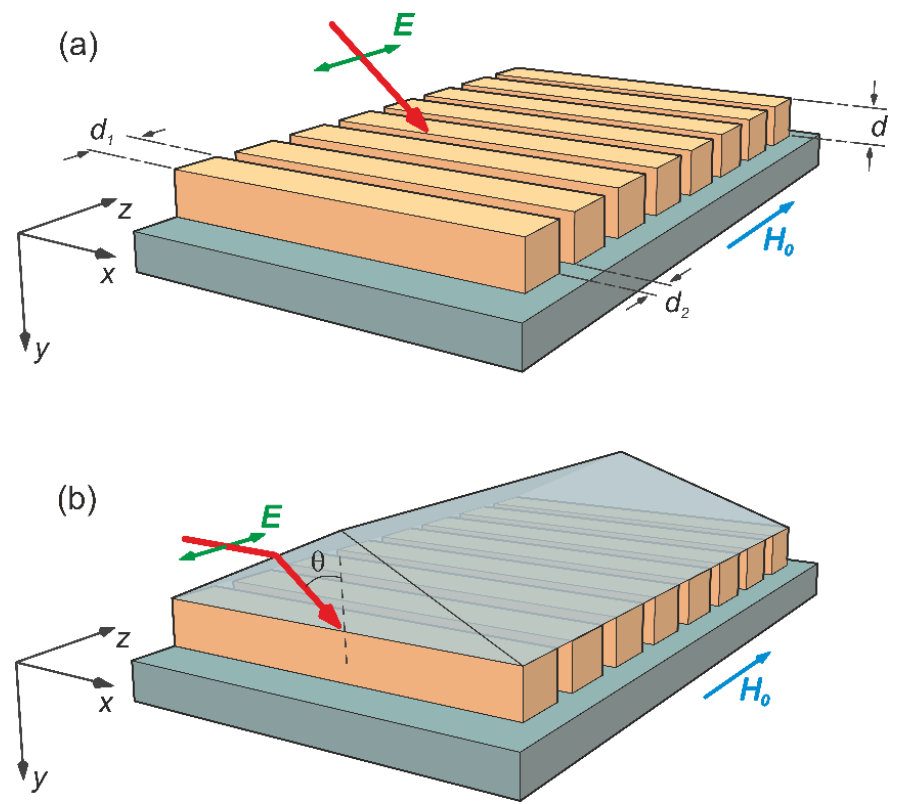

Figure 1. (a) Geometry of a nanostructured magnetic film of thickness $d$, where the individual stripes have width $d_{1}$ and spaced by $d_{2}$. The geometry of the incident electromagnetic wave is also shown; a TE-polarized incident beam whose E-field is directed along $z$ and perpendicular to the structuring direction. (b) Illustration of the Kretschmann ATR geometry with the magnetic grating, static applied 
field $H_{0}$, and the coordinate axes. The incident wave (also TE-polarized) is at an angle $\theta$ with respect to the surface normal inside a dielectric prism.

The width of the individual metallic strips is $d_{1}$ and the width of the dielectric strips is $d_{2}$. The parameters of the nanostructured metal film include a thickness $(d)$ and the period of the grating $\left(\Lambda=d_{1}+d_{2}\right)$. The dielectric tensors for the dielectric strips and dielectric substrate are diagonal and have relative values $\varepsilon_{d}$, and $\varepsilon_{s}$, respectively. The dielectric tensor for the metal film composing the grating is also diagonal and is denoted by $\varepsilon_{m}$.

We can regard the nanostructured metal films as an effective medium when the period of the one-dimensional nanostructures is smaller than a wavelength or a decay length. In the effectivemedium approximation, the graded material (the nanostructured metal film) is replaced by an effective medium having a permittivity tensor which depends upon the fraction of the material which is dielectric versus metallic. The resulting ATR reflectance calculations are straightforward. However, the effective-medium approximation makes use of the assumption that the amplitudes of the $\mathbf{E}$ and $\mathbf{B}$ fields do not vary substantially across an individual material. We note that this approximation eliminates any features that arise from the periodicity of the material including the introduction of band gaps or the shifting of wave vectors by reciprocal lattice vectors. Nonetheless, this approximation can work quite well as we showed in a previous work comparing effective medium grating results to those found for a full expansion of the periodic solutions in each region. [32]

An effective-medium approximation is used, appropriate for the orientation of the electric field component being perpendicular with respect to the grating in the metal film. The dielectric constants for the metal and the dielectric spacers in the grating are $\varepsilon_{m}$ and $\varepsilon_{d}$, respectively. The effective medium dielectric tensor, $\overleftrightarrow{\varepsilon}(\omega)$, appropriate for the geometry in Figure 1 is given by:

$$
\overleftrightarrow{\varepsilon}(\omega)=\left(\begin{array}{ccc}
\varepsilon_{x x} & 0 & 0 \\
0 & \varepsilon_{y y} & 0 \\
0 & 0 & \varepsilon_{z z}
\end{array}\right)=\left(\begin{array}{ccc}
f_{m} \varepsilon_{m}+f_{d} \varepsilon_{d} & 0 & 0 \\
0 & f_{m} \varepsilon_{m}+f_{d} \varepsilon_{d} & 0 \\
0 & 0 & \left(\frac{f_{m}}{\varepsilon_{m}}+\frac{f_{d}}{\varepsilon_{d}}\right)^{-1}
\end{array}\right)
$$

where $f_{m}$ is the fraction of the film made up of the metallic magnetic material and is given by: 


$$
\mathrm{f}_{\mathrm{m}}=\frac{\mathrm{d}_{1}}{\mathrm{~d}_{1}+\mathrm{d}_{2}}
$$

Similarly, $f_{d}$ is the fraction of the film made up of the dielectric material and is given by:

$$
\mathrm{f}_{\mathrm{d}}=\frac{\mathrm{d}_{2}}{\mathrm{~d}_{1}+\mathrm{d}_{2}}
$$

The frequency dependent magnetic permeability tensor for the metallic material has the form:

$$
\overleftrightarrow{\mu}(\omega)=\left(\begin{array}{ccc}
\mu_{\mathrm{xx}} & \mu_{\mathrm{xy}} & 0 \\
\mu_{\mathrm{yx}} & \mu_{\mathrm{yy}} & 0 \\
0 & 0 & 1
\end{array}\right)
$$

with the relevant components for a typical ferromagnetic material listed in the Methods section. The dielectric fill material and the substrate are both non-magnetic and have a permeability of unity. The permeability tensor for the effective medium representing the nanostructured metal film is:

$$
\overleftrightarrow{\mu}(\omega)=\left(\begin{array}{ccc}
\mu_{1} & i \mu_{2} & 0 \\
-i \mu_{2} & \mu_{1} & 0 \\
0 & 0 & 1
\end{array}\right)=\left(\begin{array}{ccc}
f_{m} \mu_{x x}+f_{d} & f_{m} \mu_{x y} & 0 \\
f_{m} \mu_{y x} & f_{m} \mu_{y y}+f_{d} & 0 \\
0 & 0 & 1
\end{array}\right)
$$

\subsection{Attenuated Total Reflection off a Magnetic grating}

The ATR geometry used to probe the electromagnetic response of the magnetic grating is illustrated in Figure 1(b). A high index of refraction prism, with permittivity denoted by $\varepsilon_{p}$, is used in the Kretschmann configuration [33] to increase the momentum of the incident photons. The structured metallic film is placed on the base of the prism with no intermediate layer between the film and prism. We also consider that the film is grown on a dielectric substrate with permittivity $\varepsilon_{S}$. The incident electromagnetic radiation has an angular frequency $\omega$, and electric field is in the $z$-direction and always parallel to the surface and perpendicular to the nanobars.

To find the reflectivity coefficient, one first assumes a wave-like behavior in each region. We assume that the incident wave is transverse electric (TE) polarized, with $\mathbf{E}$ directed along the $z$ direction. Thus, the $\mathbf{E}$-field in each of the three regions can be written as

$$
\mathbf{E}_{\mathbf{1}}(\boldsymbol{x}, \mathrm{t})=\left[a_{1} \mathrm{e}^{\mathrm{i} \mathrm{k}_{1 \perp}(\mathrm{y})}+b_{1} \mathrm{e}^{-\mathrm{i} \mathrm{k}_{1 \perp}(\mathrm{y})}\right] \mathrm{e}^{\mathrm{i}\left(\mathrm{k}_{\|} \mathrm{x}-\omega \mathrm{t}\right)} \hat{\mathrm{z}}
$$

in the prism (first region), 


$$
\mathbf{E}_{2}(\boldsymbol{x}, \mathrm{t})=\left[a_{2} \mathrm{e}^{\mathrm{i} \mathrm{k}_{2 \perp}(\mathrm{y})}+\boldsymbol{b}_{2} \mathrm{e}^{-\mathrm{i} \mathrm{k}_{2 \perp}(\mathrm{y})}\right] \mathrm{e}^{\mathrm{i}\left(\mathrm{k}_{\|} \mathrm{x}-\omega \mathrm{t}\right)} \hat{\mathrm{z}}
$$

in the effective medium (second region), and

$$
\mathbf{E}_{3}(\mathbf{x}, \mathrm{t})=a_{3} \mathrm{e}^{\mathrm{i} \mathrm{k}_{3 \perp}(\mathrm{y})} \mathrm{e}^{\mathrm{i}\left(\mathrm{k}_{\|} \mathrm{x}-\omega \mathrm{t}\right)} \hat{\mathrm{z}}
$$

in the substrate (third region).

The parallel component of the wave vector, $k_{\|}=\frac{\omega}{c} \sqrt{\varepsilon_{p}} \sin \theta$, is constant in all three regions and the perpendicular components are determined by using the dispersion relation for each medium separately. One finds

$$
\begin{gathered}
k_{1 \perp}^{2}=\left(\frac{\omega}{c}\right)^{2} \epsilon_{p}-k_{\|}{ }^{2}, \\
k_{2 \perp}^{2}=\left(\frac{\mu_{1}{ }^{2}-\mu_{2}{ }^{2}}{\mu_{1}}\right)\left(\frac{\omega}{c}\right)^{2} \epsilon_{z z}-k_{\|}{ }^{2},
\end{gathered}
$$

and

$$
k_{3 \perp}^{2}=\left(\frac{\omega}{c}\right)^{2} \epsilon_{d}-k_{\|}^{2}
$$

To find the reflectance, one must also satisfy the boundary conditions at each surface. We thus require that $H_{x}$ and the tangential component of $\boldsymbol{E}$ be continuous at the interfaces. Since we know the E-field from Equation (6)-(8), the $H$-field associated with each of the electric fields is found by applying Maxwell's equations. By use of $\boldsymbol{\nabla} \times \boldsymbol{E}=-\frac{\partial \boldsymbol{B}}{\partial t}$ and $\boldsymbol{B}=\mu_{0} \overleftrightarrow{\mu}(\omega) \boldsymbol{H}$ a general relationship between $E$ and $H_{x}$ for this problem is found to be:

$$
H_{x}(\boldsymbol{x}, t)=\frac{c}{i \omega\left(\mu_{1}^{2}-\mu_{2}^{2}\right)}\left[\mu_{1} \frac{\partial E}{\partial y}+i \mu_{2} \frac{\partial E}{\partial x}\right] .
$$

Note that this is the most general case - that of the structured grating where $\overleftrightarrow{\mu}(\omega)$ is given by Equation (5). However, Equation (12) also applies to the other layers; as these layers are dielectric, for them we can simply make $\mu_{1}=1$ and $\mu_{2}=0$. Continuity of tangential $\mathrm{E}$ and $\mathrm{H}_{\mathrm{x}}$ at $y=0$ (the boundary between the prism and the effective medium grating so that $\mathbf{E}_{\mathbf{1}}=\mathbf{E}_{\mathbf{2}}$ and $\mathrm{H}_{1 \mathrm{x}}=\mathrm{H}_{2 \mathrm{x}}$ ) yield the following two equations:

$$
a_{1}=-b_{1}+a_{2}+b_{2}
$$


and

$$
a_{1} k_{1 \perp}=b_{1} k_{1 \perp}+a_{2} k_{+}+b_{2} k_{-}
$$

where

$$
k_{ \pm}=\frac{i k_{x} \mu_{2} \pm k_{2 \perp} \mu_{1}}{\mu_{1}^{2}-\mu_{2}^{2}}
$$

Similarly, continuity of $\mathbf{E}$ and $\mathrm{H}_{\mathrm{x}}$ at $y=d$ (the boundary between the effective medium grating and the substrate so that $\mathbf{E}_{2}=\mathbf{E}_{3}$ and $\mathrm{H}_{2 \mathrm{x}}=\mathrm{H}_{3 \mathrm{x}}$ ) results in the two following equations:

$$
a_{2} e^{i k_{2 \perp} d}+b_{2} e^{-i k_{2 \perp} d}-a_{3} e^{i k_{3 \perp} d}=0,
$$

and

$$
a_{2} k_{+} e^{i k_{2 \perp} d}+b_{2} k_{-} e^{-i k_{2 \perp} d}-a_{3} k_{3 \perp} e^{i k_{3 \perp} d}=0 .
$$

This system of four-equations and four-unknowns is easily solved numerically for the reflectance by setting the variable $a_{1}$ equal to one and finding the ATR signal by $\left|b_{2}\right|^{2}$.

\section{RESULTS}

Unless otherwise indicated, the parameters for our calculations are as follows. The dielectric medium that fills the grating nanoslits has an index of refraction of 1.333. This value is typical of water as might be found in a biosensing application, but this is also a typical value for Teflon which is widely employed in microwave devices [34]. There is a dielectric medium below the film again with $\mathrm{n}=1.333$ - corresponding to $\varepsilon_{d}=\varepsilon_{s}=n^{2}$. We consider Transverse Electric (TE) polarized incident light with frequencies that varies from $3 \mathrm{GHz}$ to $350 \mathrm{GHz}$ at incident angle $\theta$ and we take the speed of light to be $c=3.0 \times 10^{10} \mathrm{~cm} \mathrm{sec}^{-1}$. The refractive index of the prism is 1.723 (SF10 glass) [35] and the electrical permittivity of the metal is frequency dependent: $\varepsilon_{m}=1+\frac{i \sigma}{\varepsilon_{0} \omega}$; with $\sigma=$ $1.02 \times 10^{7} \mathrm{~S} \mathrm{~m}^{-1}$, which is appropriate for iron, and the permittivity of free space is $\varepsilon_{0}=$ $8.85 \times 10^{12} \mathrm{~F} \mathrm{~m}^{-1}$. 
Before detailing our main findings, we first note that for our geometry for reflectivity we only require $\varepsilon_{z z}$ [see Eq. (1)], which from here onwards we will simply refer to as the effective dielectric constant of the grating structure, $\varepsilon_{e f f}$. This constant varies significantly with the filling factors following the relation

$$
\frac{1}{\varepsilon_{e f f}}=\frac{f_{m}}{\varepsilon_{m}}+\frac{f_{d}}{\varepsilon_{d}}
$$

But since the metal has a very high epsilon, this reduces to a simple equation where

$$
\varepsilon_{e f f} \approx \frac{\epsilon_{d}}{f_{d}}
$$

A critical value of $f_{d}$ occurs when $\varepsilon_{e f f}>\varepsilon_{p}$. Values above this are in the "fiber optic" limit in the sense that the grating region has a higher index of refraction compared to the surrounding regions of the prism or substrate. (We note that having an index of refraction in a glass-like fiber that is larger than the index of refraction in the surrounding material leads to total internal reflection of an electromagnetic wave in the fiber with an evanescent wave outside the fiber $[36,37]$. We will see similar behavior in our system.) Substituting the parameters listed above, this gives

$$
f_{d}=\frac{\varepsilon_{d}}{\varepsilon_{p}}=\frac{1.776}{2.968}=0.60,
$$

and the critical value for the metal filling fraction is $f_{m}=0.40$.

\subsection{Reflectivity off an all-dielectric structure}

To create the proper context, we find it useful to first investigate the case where there is no magnetic response in the grating. For this, we examine results for ATR-like measurements from a purely dielectric structure such as that shown in Figure 1(b), when the relative magnetic permeability tensor is artificially set to the unit matrix. In Figure 2(a) we show the ATR results, color maps of the reflectance as a function of incident angle and frequency, for the purely dielectric structure (prism/grating structure/substrate) where the metallic portion of the grating is nonmagnetic. The metallic filling fraction in the grating structure is $f_{m}=0.61$, and the thickness of the effective medium film is $0.1 \mathrm{~cm}$. Furthermore, the dielectric materials are, initially, taken to have purely real 
epsilon values. We see two distinct regions: a reflectivity of unity (red) for angles beyond $\theta=50.6$ degrees, and a low reflectivity for angles below 50.6 degrees. If one calculates the critical angle between the high-index prism and the low index substrate, it is found to be $50.6^{\circ}$ [this is shown as the vertical dashed lines in Figure 2(a)-(b)]. For angles below this value, one does not have total internal reflection and hence the central region in the ATR curves generally corresponds to lowered transmission.
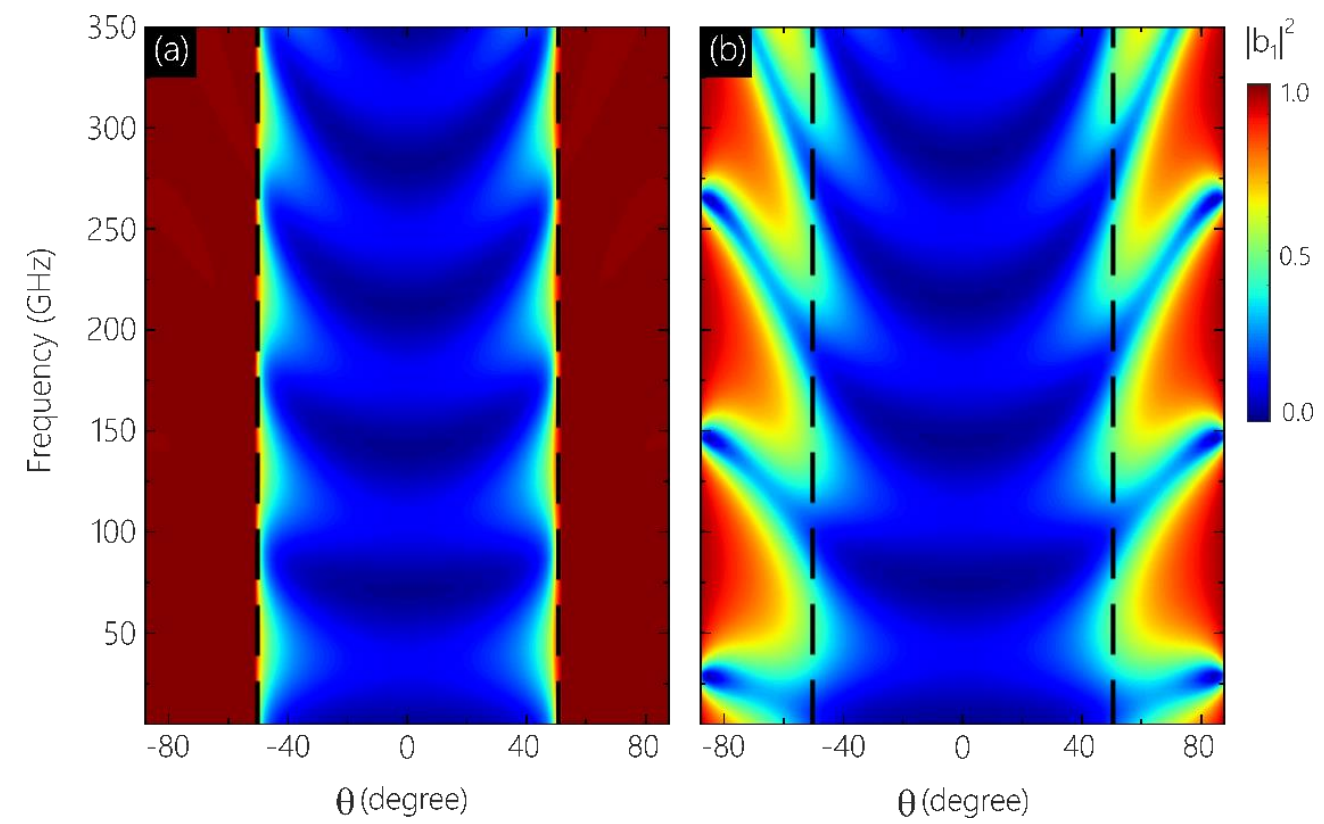

Figure 2. (a) ATR spectra maps showing the reflectance as a function of incident angle and frequency. The overlayer has a dielectric constant of $\varepsilon_{p}=2.968$ and the substrate's is $\varepsilon_{s}=1.33$. The middle layer takes the effective dielectric constant of the metallic grating, $\varepsilon_{\text {eff }}$, albeit being nonmagnetic [so that $\overleftrightarrow{\mu}(\omega)=1]$. Here, $f_{m}=0.61$, and $d=0.1 \mathrm{~cm}$. Note that all three dielectric constants are purely real and in (b) we show the effect of damping by making the dielectric constant of the substrate $\varepsilon_{3}=$ $1.33+0.5 i$

Figure 2(b) expands the situation by adding a small imaginary portion to the dielectric constant of the substrate material allowing the incident radiation to couple to surface modes, i.e. $\varepsilon_{s}=1.333^{2}+$ $0.5 i$. In addition to the features described above, there are now narrow fingers of reduced reflectivity extending beyond the central region. As we will see later, these correspond to fiber optic like states 
where the grating region has a higher index of refraction compared to the surrounding regions of the prism or substrate [36]. Much like surface polaritons, these fiber optic like states (also known as guided waves) interact with the incident wave only at certain frequencies where they can exist—here related to the thickness on the film, or its dielectric constant—so that the reflectivity dips almost to zero. Basically, in the regions where the fiber optic states can exist, they carry off the energy of the incident radiation, resulting in a reduction in the reflectivity. We note that all of the results are strictly reciprocal, i.e. the reflectivity for the positive and negative values of the incident angle is identical.

\subsection{Reflectivity off a magnetic grating}

In Figure 3 we show results for the case where the magnetic response in the grating is turned back on, but we keep the same dielectric parameters as those used in Figure 2(b). There are substantial differences between Figure 2(b) and Figure 3. First, all the reflectance map is now significantly nonreciprocal. This is most evident in the frequency range of $30-80 \mathrm{GHz}$, where there is a distinct finger-like feature for negative angles, and no corresponding feature on the right. However, there are other nonreciprocal features as well. If we look at the black-dashed horizontal line marking the 150 GHz position, it is easy to see that the results for positive and negative angles are not the same, with the features on the left side being shifted up by about $10 \mathrm{GHz}$ compared to the similar features on the right side. As one goes higher in frequency, the nonreciprocity becomes smaller, but is still present throughout. 


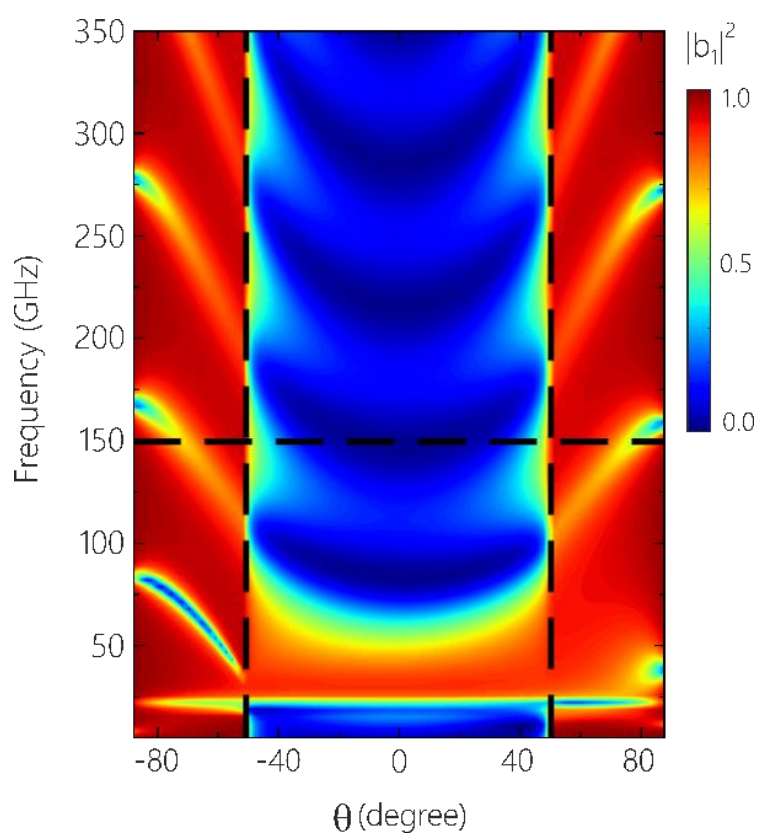

Figure 3. ATR spectra map for the same dielectric parameters as 3(b) except now the magnetic permeability is also included [according to Eq. (5)]. We note the distinct nonreciprocity in the reflectivity for $|\theta|>50^{\circ}$ at frequencies ranging from about $30-80 \mathrm{GHz}$. Using the horizontal black line, as a guide, at $150 \mathrm{GHz}$ we can see there is also substantial nonreciprocity even at that frequency.

In Figure 4 we explore how the ATR behavior changes as a function of the magnetic filling fraction, $\mathrm{f}_{1}$. There are several interesting features which can be seen in Figure 4:

1) Again, there is a central region $\left(|\theta|<50.6^{\circ}\right)$ where the reflectivity is low, corresponding to angles below the critical angle as described above. The exceptions to this are where the magnetic material has a stop band, i.e. a frequency region where no electromagnetic waves propagate. In this case the frequency range of the stop band depends on the filling fraction. At low filling fractions the stop-band is restricted to values near $f=\mu_{0} \gamma \sqrt{H(H+M)}=29 \mathrm{GHz}$, for larger values of the filling fraction the magnetic stop band extends up to the so-called antiresonance frequency [38] of $f=\mu_{0} \gamma(H+M)=74.5 \mathrm{GHz}$, for the parameters used here.

2) For some filling fraction values there is a strong non-reciprocity, in reflection that extends from about $25 \mathrm{GHz}$ to over $120 \mathrm{GHz}$. 
3) The distinctive bands of non-reciprocity are primarily seen at incident angles above approximately 50-degrees; although some non-reciprocal behavior also seen at smaller incident angle for some frequency ranges.

4) At low filling fractions, there is a large region where the reflectivity is nearly zero. As the filling fraction increases, this breaks up into individual bands of low reflectivity. This is primarily due to the dielectric response of the medium as indicated in Figure 2.

5) We should also mention that the positions of the non-reciprocal features are tunable with the applied magnetic field. For example, decreasing the external field from $0.6 \mathrm{~T}$ to $0.2 \mathrm{~T}$ shifts the non-reciprocal ranges down in frequency by $10 \mathrm{GHz}$.
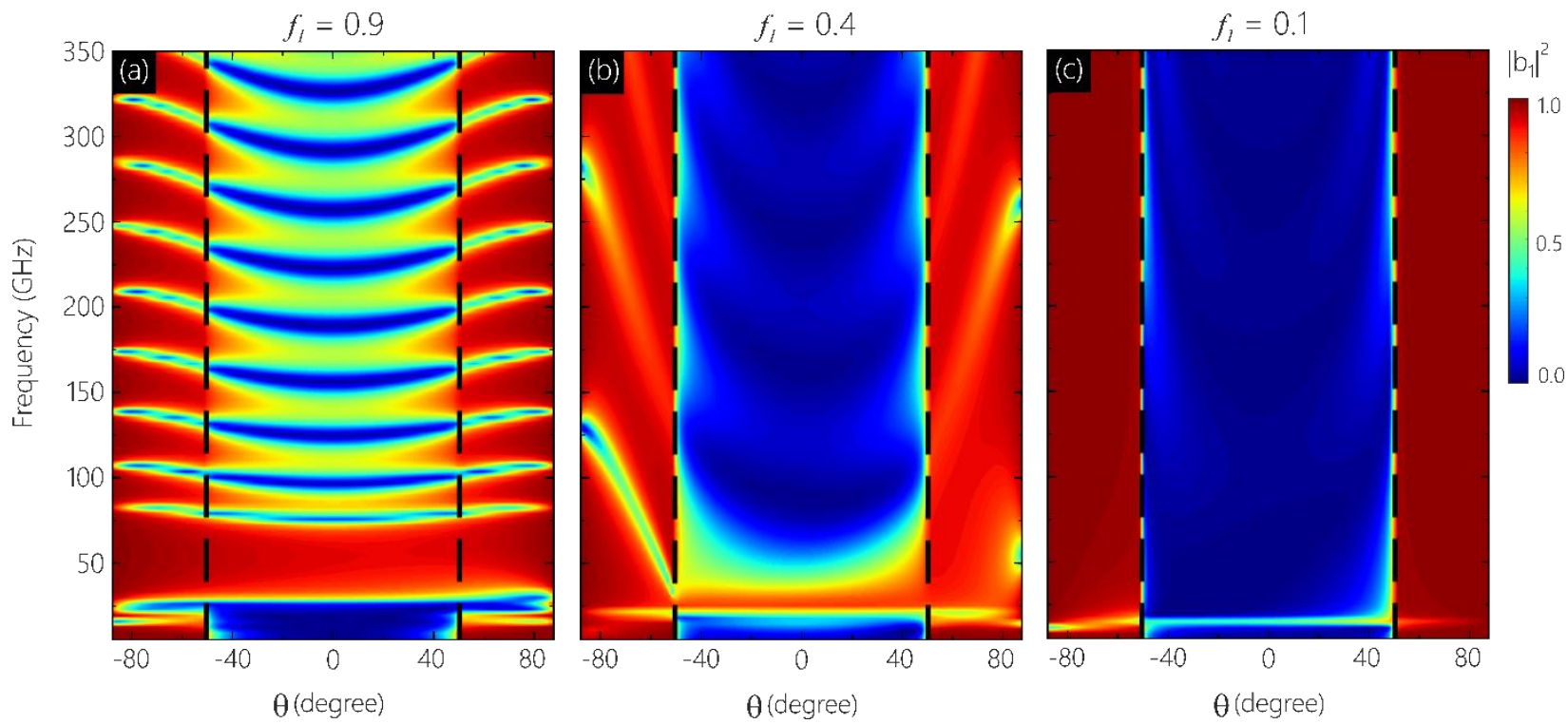

Figure 4: ATR spectra maps for different values of the filling factor, $f_{1}$. The color bar on the right gives the reflectance values, which in the maps are plotted as a function of both the incident angle and the frequency. The specific parameters for these plots are $\mu_{0} H_{0}=0.4 T, d=0.1 \mathrm{~cm}, \alpha=0.02$. The dielectric parameters for all layers are those of Figure 2(b). 


\subsection{Dispersion Relation for Bound Polaritons}

To understand the ATR results, it is helpful to calculate the polariton modes that exist in our effective medium film surrounded by two different dielectrics, as shown in Figure 5. The investigation of the TE-polarized polaritons is done by determining the dispersion relation in the film and the surrounding dielectric materials, which is then used with the surface boundary conditions to determine the wave profiles for the bound polaritons. Because this calculation is similar to the ATR calculation described earlier, we only give a brief outline.

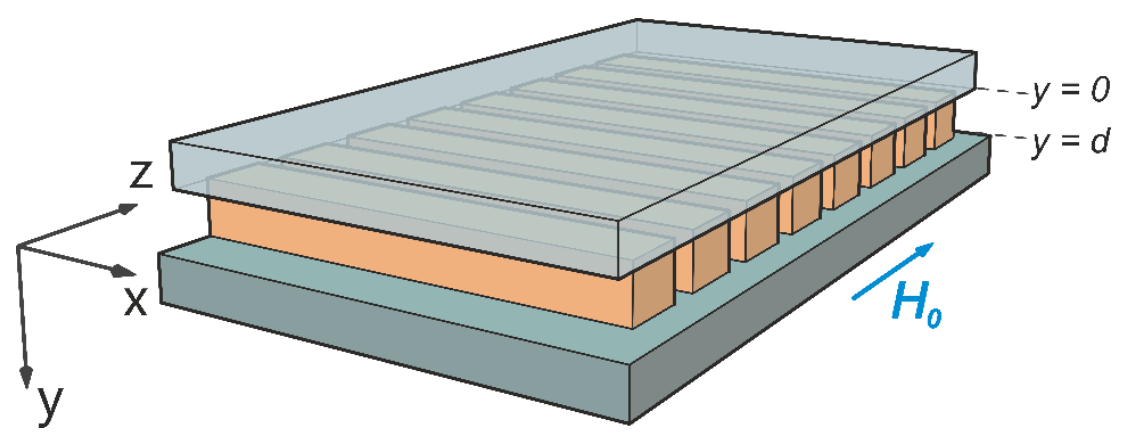

Figure 5: Geometry used for the calculation of the dispersion relation for bound polaritons.

Again, one first assumes a wave-like behavior in each region around the film, with the E-field only in the z-direction as shown in Figure 2. The electric fields are given by

$$
\begin{array}{cl}
\boldsymbol{E}(\boldsymbol{x}, t)=\mathcal{A} e^{+\alpha(y)} e^{i\left(k_{\|} x-\omega t\right)} \hat{\mathrm{z}}, & \text { for } \mathrm{y}<0 \\
\boldsymbol{E}(\boldsymbol{x}, t)=\left[\mathcal{B} e^{i k_{2 \perp}(y)}+\mathcal{C} e^{-i k_{2 \perp}(y)}\right] e^{i\left(k_{\|} x-\omega t\right)} \hat{\mathrm{z}}, & \text { for } 0<\mathrm{y}<\mathrm{d}
\end{array}
$$

and

$$
\boldsymbol{E}(\boldsymbol{x}, t)=\mathcal{D} e^{-\beta(y)} e^{i\left(k_{\|} x-\omega t\right)} \hat{\mathrm{z}} . \quad \text { for } \mathrm{y}>\mathrm{d}
$$

The values of $\alpha$ and $\beta$ can be obtained from Equation (9) and Equation (11) by making $\alpha=i k_{1 \perp}$ and $\beta=i k_{3 \perp}$ and we assume that the real part of $\alpha$ and $\beta$ are positive.

To find the dispersion of the polariton, one must also satisfy the boundary conditions at each surface. We thus require that $H_{x}$ and the tangential component of $\boldsymbol{E}$ be continuous at the interfaces. Continuity of tangential $\boldsymbol{E}$ at $y=0$ and $y=+d$ gives the following two equations: 


$$
\mathcal{A}-\mathcal{B}-\mathcal{C}=0
$$

and

$$
\mathcal{B} e^{i k_{2 \perp} d}+\mathcal{C} e^{-i k_{2 \perp} d}-\mathcal{D} e^{-\beta d}=0
$$

Similarly, continuity of $\mathrm{H}_{\mathrm{x}}$ at each of the boundaries results in the two following equations:

$$
k_{1 \perp} \mathcal{A}-k_{-} \mathcal{B}+k_{+} \mathcal{C}=0
$$

and

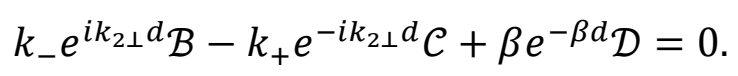

again where $k_{ \pm}$is given by Equation (15).

Equation (24) to Equation (27) are a homogeneous set of equations for the variables $\mathcal{A}, \mathcal{B}, \mathcal{C}$, and $\mathcal{D}$. When put in matrix form, the determinant matrix, $\mathcal{M}$, must be zero for a nontrivial solution. The solutions can be found in a number of ways. First one can plot the $\log (|\boldsymbol{M C}|)$ as a function of $\operatorname{Re}\left(k_{\|}\right)$and $\omega$ as a color map. Smaller values for $\log (|\mathcal{M}|)$ give the positions for the dispersion relation of bound polaritons of this three-layer system. To obtain the exact values, i.e. both $\operatorname{Re}\left(k_{\|}\right)$and $\operatorname{Im}\left(k_{\|}\right)$ one must use a complex root finder.

To find the wave profiles, one uses the values of frequency and wave-vector in the boundary conditions to find, for example $\mathcal{A}, \mathcal{B}$, and $\mathcal{C}$ in terms of $\mathcal{D}$. This allows one to plot the wave profile for different locations along the dispersion curve.

To place the results for the magnetic material in context, we again start by presenting the numerical results for the dispersion relation for the dielectric-only version of this problem (so $H_{0}=0$ in Figure 5, and the permeability tensor is again set to the unit matrix). The parameters here are the same as those in Figure 3, namely $f_{m}=0.61$ and $\varepsilon_{s}=1.333^{2}+0.5 \mathrm{i}$.

The dispersion relations and mode profiles for several bound states are shown in Figure 6. In part (a), the dashed black lines indicate the boundary for what features are visible in the ATR measurement, i.e. incident angles less than $90^{\circ}$, with dispersion curves inside (lower angles) the boundary line visible in ATR and those outside are not seen. The solid black lines, and respective shaded region in the center, indicate the region where propagation should take place as the 
combination of incident angle and dielectric constant of the substrate do not allow for confinement of waves. The results agree well with what is seen in the ATR spectrum of Figure 2(b) where there are narrow regions of low reflectivity, at higher angles, from $20-30 \mathrm{GHz}$ and again for frequencies of 100 $160 \mathrm{GHz}$ and just above $250 \mathrm{GHz}$. These low reflectivity regions can, in fact, be traced to the modes (white solid lines) as the frequencies where each mode meets the prism's light line (dashed black line). In other words, the incident radiation is being effectively transferred to a surface or bound polariton, with a resultant loss in reflectivity.

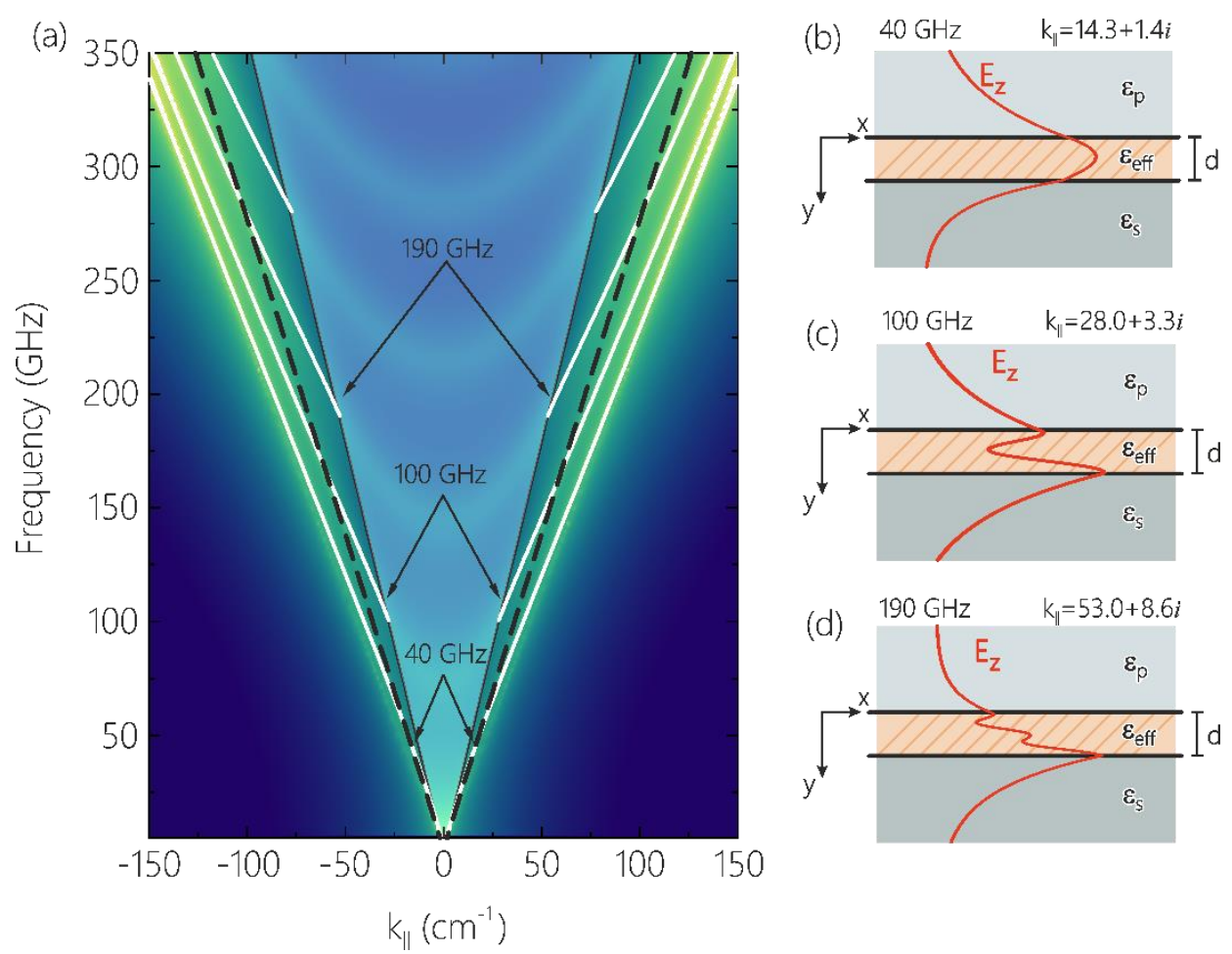

Figure 6. (a) Dispersion relations for bound polariton states in the three-layer geometry when the effective medium is not magnetic. The color map presents the $\log (|\mathcal{M}|)$ as a function of $\operatorname{Re}\left(k_{\|}\right)$and $\omega$ as discussed in the text. (b) - (d) Profiles of guided waves at different frequencies.

We can obtain insight into the nature of the modes by plotting the profiles of the various modes, as seen in Fig. 6(b)-(d). The modes have an oscillator behavior in the central region (grating) but the wave decay to zero outside that region. We note that the waves are reciprocal in that the profiles for $+k$ and $-k$ are identical. 
When the magnetic response in the grating is included, the dispersion relations change significantly as is expected from the ATR results in Figure 3 and as seen in Figure 7. The dispersion relations for the lowest frequency modes show substantial nonreciprocity, with values for the $-k$ side lying inside the region probed by ATR and the values for the $+k$ side lying mostly outside the boundary. This is exactly the behavior found in Figure 3 where there is strong nonreciprocity for the features seen in the $30-70 \mathrm{GHz}$ range.
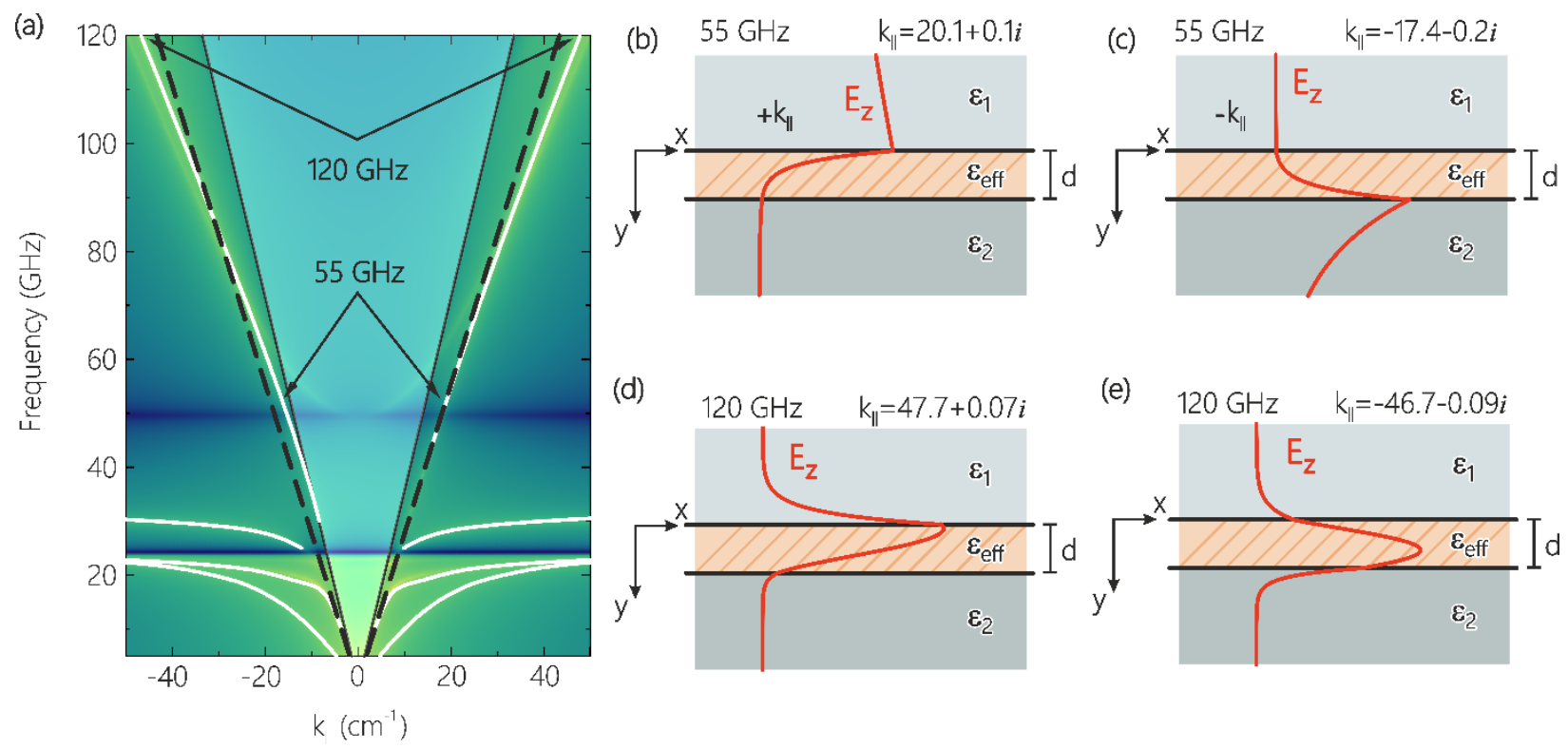

Figure 7. (a) Dispersion relations for bound polariton states in the three-layer geometry with the magnetic metallic material. The color map presents the $\log (|\mathbf{M}|)$ as a function of $\operatorname{Re}\left(k_{||}\right)$and $\omega$ as discussed in the text. (b)-(e) polariton profiles for different.

A surprising feature of the ATR and dispersion relation results is the large frequency range where there is significant nonreciprocal behavior. For magnetostatic waves nonreciprocity occurs as a result of the off-diagonal terms in the permeability, $\mu_{x y}$ or $\mu_{y x}$ [39]. Furthermore, for magnetostatic waves, nonreciprocity is confined to the frequency region where $\mu_{x x}$ is negative because for these frequencies bulk waves are forbidden [40], and one finds nonreciprocal surface waves. In contrast, in the fully-retarded electromagnetic system discussed here, we find that surface waves, or bound states, appear even in the absence of a magnetic material, as is seen in Figs 2 and 6. Therefore, there is no 
requirement for the diagonal component of the permeability tensor to be negative in order to have surface-like waves. However, a non-zero value for the off-diagonal components of the permeability is still required to have nonreciprocity.

In Figure 7 (b) and (c) we show the mode profiles at $55 \mathrm{GHz}$ which are drastically different from those of the purely dielectric case (in Figure 6). Here the bound waves are evanescent inside the grating as well as outside - which is distinct feature of true surface waves, in this case, surface magnon-polaritons. For the parameters used here, the region where these modes are found extends from region extends from about $25 \mathrm{GHz}$ to $50 \mathrm{GHz}$.

We find interesting to note however, that these surface modes, seem to continue to exist at high frequencies (looking at the white solid lines starting around 30 to $40 \mathrm{GHz}$, they continue on towards the 100 s of GHz). While it looks like the same mode, its character changes as it moves off the region where the permeability is negative. In Figure 7(d) and (e) we show its behavior at $120 \mathrm{GHz}$, where a distinct oscillating behavior is now seen inside the material.

As we have stated, the nonreciprocity is not confined to the region where the permeability is negative. As we have seen in the reflectivity, we get large nonreciprocity over a much larger frequency region, extending easily up to $300 \mathrm{GHz}$. This is still an effect of the off-diagonal component of the permeability which albeit small, it is still present up to and beyond $300 \mathrm{GHz}$. We should note that the nonreciprocity in the dispersion relations is reduced however, as the frequency increases and the effect of the off-diagonal components become less dominant. We have omitted the dispersion curves for higher frequencies for the magnetic grating as it very similar to that shown in Figure 6, but nonetheless the is a significant difference in the dispersion relations which is manifested in a nonreciprocal reflection seen in Figure 3.

\section{DISCUSSION AND CONCLUSIONS}

We have demonstrated nonreciprocal behavior over a large frequency range, extending up to $300 \mathrm{GHz}$, will occur in attenuated total reflection measurements with a magnetic grating as the active 
element. The grating is composed of a moderately thick magnetic metallic film, separated by thin insulating sections. This reduces the conductivity of the overall structure, and we obtain strong nonreciprocal electromagnetic responses at high frequencies.

A way to realise the structures that we discuss here, is to use Focused Ion Beam (FIB) techniques which can be used to cut, slice, and drill magnetic samples [41, 42]. For effective medium theory to be appropriate, one wants the overall change in the fields to be small over the length of a unit cell. In this problem, the metallic skin depth - typically on the order of a few microns—sets the length scale for the size of a unit cell. Because FIB systems can make cuts, with dimensions as low a few nanometers, it should be possible to fabricate appropriate unit cells with a variety of fill factors.

We show that the frequency regions of high absorption and significant nonreciprocity can be substantially modified by changing the filling fraction of the grating. By calculating the dispersion relations of the magnetic polariton modes of an insulator/grooved metal/ insulator structure and connecting their spatial profiles to the nonreciprocal reflectivity results we are able to understand the reflectivity results. The drivers for the nonreciprocal behavior are the off-diagonal components of the permeability tensor which are present up to and beyond $300 \mathrm{GHz}$, and the presence of surface (or bound polariton) modes which result from the dielectric properties of the grating.

These findings could be verified experimentally through a variety of techniques, including standard reflectivity or ATR measurements. For example, reflectivity experiments in the 200-300 GHz range have been developed for antiferromagnetic crystals where the incident radiation is created by a third harmonic wave generated from an impatt diode with fundamental frequency at $88 \mathrm{GHz}$. [43]. At lower frequencies, we expect that the metamaterial should be easy to incorporate into microwave waveguide structures where the electromagnetic behaviour can be probed. The lowered conductivity should lead to much stronger response than that of conventional, continuous metallic films. Finally, it is noted that, in addition to the magnetic material filling fraction and overall metamaterial thickness, the positions of the non-reciprocal features can be tuned with the applied magnetic field. 


\section{METHODS}

Permeability tensor and parameters for the magnetic material: The permeability tensor for the magnetic material is given by the following:

$$
\mu=\left(\begin{array}{ccc}
\mu_{x x} & \mu_{x y} & 0 \\
\mu_{y x} & \mu_{y y} & 0 \\
0 & 0 & 1
\end{array}\right) .
$$

Here

$$
\mu_{x x}=\mu_{y y}=1+\frac{\omega_{M}\left(\omega_{H}\left(1+\alpha^{2}\right)-i \omega \alpha\right)}{\omega_{H}^{2}\left(1+\alpha^{2}\right)-2 i \omega \alpha \omega_{H}-\omega^{2}}
$$

and

$$
\mu_{x y}=-\mu_{y x}=i \frac{\omega_{M} \omega}{\omega_{H}^{2}\left(1+\alpha^{2}\right)-2 i \omega \alpha \omega_{H}-\omega^{2}}
$$

The terms in these equations are:

$$
\begin{aligned}
& \omega_{M}=\gamma \mu_{0} M \\
& \omega_{H}=\gamma \mu_{0} H_{0}
\end{aligned}
$$

Here the gyromagnetic ratio is $\gamma=2 \pi \times 29 G H z T^{-1}$. The magnetization, $\mu_{0} \mathrm{M}=2.17 \mathrm{~T}$, appropriate for iron, and the applied field, $\mu_{0} H_{0}=0.4 \mathrm{~T}$ unless otherwise noted. The Gilbert damping parameter, $\alpha$, has the values denoted in the text.

\section{REFERENCES}

[1] C. L. Hogan, The Ferromagnetic Faraday Effect at Microwave Frequencies and its Applications: The Microwave Gyrator, Bell Syst. Tech. J. 31, 1-31 (1952). doi: 10.1002/j.15387305.1952.tb01374.x.

[2] C. L. Hogan, The ferromagnetic faraday effect at microwave frequencies and its applications, Rev. Mod. Phys. 25, 253-262, (1953). doi: 10.1103/RevModPhys.25.253.

[3] G. H. B. Thompson, Unusual Waveguide Characteristics associated with the Apparent Negative Permeability obtainable in Ferrites, Nature 175, 1135-1136 (1955). https://doi.org/10.1038/1751135b0 
[4] R. Zivieri, Dynamic negative permeability in a lossless ferromagnetic medium, 9th International Congress on Advanced Electromagnetic Materials in Microwaves and Optics (METAMATERIALS), 532-534, (2015). doi: 10.1109/MetaMaterials.2015.7342513.

[5] R. Zivieri, Dynamic permeability in a dissipative ferromagnetic medium, 10th International Congress on Advanced Electromagnetic Materials in Microwaves and Optics (METAMATERIALS), 427-429, (2016). doi: 10.1109/MetaMaterials.2016.7746424.

[6] B. K. Kuanr, V. Veerakumar, R. Marson, S. R. Mishra, R. E. Camley, and Z. Celinski, Nonreciprocal microwave devices based on magnetic nanowires, Appl. Phys. Lett. 94, 202505 (2009). doi: 10.1063/1.3124657.

[7] V. Cherepanov, I. Kolokolov, and V. L'vov, The saga of YIG: Spectra, thermodynamics, interaction and relaxation of magnons in a complex magnet, Physics Reports 229, 81-144 (1993). doi: 10.1016/0370-1573(93)90107-O.

[8] V. G. Harris et al., Recent advances in processing and applications of microwave ferrites, $J$. Magn. Magn. Mater. 321, 2035-2047 (2009). doi: 10.1016/j.jmmm.2009.01.004.

[9] M. Giordani, M. Polese, M. Mezzavilla, S. Rangan and M. Zorzi, Toward 6G Networks: Use Cases and Technologies, IEEE Communications Magazine 58, 55-61 (2020). doi: 10.1109/MCOM.001.1900411.

[10] T. Huang, W. Yang, J. Wu, J. Ma, X. Zhang and D. Zhang, A Survey on Green 6G Network: Architecture and Technologies, IEEE Access 7, 175758-175768 (2019). doi: 10.1109/ACCESS.2019.2957648.

[11] S. Yuan, L. Chen, Z. Wang, et al. On-chip terahertz isolator with ultrahigh isolation ratios, Nat Commun 12, 5570 (2021). https://doi.org/10.1038/s41467-021-25881-0

[12] B. Heydari, M. Bohsali, E. Adabi and A. M. Niknejad, Millimeter-Wave Devices and Circuit Blocks up to $104 \mathrm{GHz}$ in $90 \mathrm{~nm}$ CMOS, IEEE Journal of Solid-State Circuits 42, 2893-2903 (2007). doi: 10.1109/JSSC.2007.908743.

[13] J. Zhao, C. Li, X. Li, and L. Shen, Technical challenges for high-frequency wireless 
communication, J. Commun. Inf. Networks 1, 19-28 (2016). doi: 10.1007/bf03391554.

[14] B. Kuanr, Z. Celinski, and R. E. Camley, Tunable high-frequency band-stop magnetic filters, Appl. Phys. Lett. 83, 3969-3971, (2003). doi: 10.1063/1.1625424.

[15] R. Macêdo, Tunable Hyperbolic Media: Magnon-Polaritons in Canted Antiferromagnets, in Solid State Physics vol. 68, edited by Robert E. Camley, Robert L. Stamps (Burlington: Academic Press, 2017), Chap. 2. pp. 91-155. https://doi.org/10.1016/bs.ssp.2017.10.001

[16] R. G. Chambers, Anomalous skin effect in metals, Nature 165, 239-240 (1950). doi: $10.1038 / 165239 b 0$.

[17] T. J. Fal and R. E. Camley, Hexagonal ferrites for use in microwave notch filters and phase shifters, J. Appl. Phys. 104, 23910 (2008). doi: 10.1063/1.2957066.

[18] Z. Wang et al., Millimeter wave phase shifter based on ferromagnetic resonance in a hexagonal barium ferrite thin film, Appl. Phys. Lett. 97, 72509 (2010). doi: 10.1063/1.3481086.

[19] Y. Y. Song, J. Das, Z. Wang, W. Tong, and C. E. Patton, In-plane c-axis oriented barium ferrite films with self-bias and low microwave loss, Appl. Phys. Lett. 93, 172503 (2008). doi: 10.1063/1.3010374.

[20] E. Salahun, P. Quéffélec, G. Tanné, A. L. Adenot, and O. Acher, Correlation between magnetic properties of layered ferromagnetic/dielectric material and tunable microwave device applications, J. Appl. Phys. 91, 5449-5455 (2002). doi: 10.1063/1.1461066.

[21] D. R. Smith, et al. Composite medium with simultaneously negative permeability and permittivity. Phys. Rev. Lett. 84, 4184 (2000). https://doi.org/10.1103/PhysRevLett.84.4184

[22] V. G. Veselago, The Electrodynamics of Substances with Simultaneously Negative Values of $\varepsilon$ and $\mu$, Sov. Phys. Usp. 10, 509 (1968). https://doi.org/10.1070/PU1968v010n04ABEH003699

[23] M. Kadic, G. W. Milton, M. van Hecke, et al. 3D metamaterials. Nat. Rev. Phys. 1, 198-210 (2019). https://doi.org/10.1038/s42254-018-0018-y

[24] T. Lv, Y. Li, H. Ma, et al. Hybrid metamaterial switching for manipulating chirality based on $\mathrm{VO}_{2}$ phase transition. Sci. Rep. 6, 23186 (2016). https://doi.org/10.1038/srep23186 
[25] Y. Xiang, X. Dai, J. Guo, et al. Critical coupling with graphene-based hyperbolic metamaterials. Sci. Rep. 4, 5483 (2014). https://doi.org/10.1038/srep05483

[26] R. Macêdo, K. L. Livesey, and R. E. Camley, Using magnetic hyperbolic metamaterials as high frequency tunable filters, Appl. Phys. Lett. 113, 1-6 (2018), doi: 10.1063/1.5049602.

[27] P. M. Yarbrough, K. L. Livesey, R. E. Camley, and R. Macêdo, Far-Infrared Reflection from Heterostructures Made of Ultrathin Ferromagnetic Layers, Phys. Rev. Appl. 12, 024004 (2019). doi: 10.1103/PhysRevApplied.12.024004.

[28] R. V. Mikhaylovskiy, E. Hendry and V. V. Kruglyak, Negative permeability due to exchange spin-wave resonances in thin magnetic films with surface pinning, Phys. Rev. B 82, 195446 (2010). https://doi.org/10.1103/PhysRevB.82.195446

[29] J. Rensberg et al., Active Optical Metasurfaces Based on Defect-Engineered Phase-Transition Materials, Nano Lett 16, 48 (2016). doi: 10.1021/acs.nanolett.5b04122.

[30] K.-P. Chen, S.-C. Ye, C.-Y. Yang, Z.-H. Yang, W. Lee, and M.-G. Sun, Electrically tunable transmission of gold binary-grating metasurfaces integrated with liquid crystals, Opt. Express 24, 16815 (2016). doi: 10.1364/oe.24.016815.

[31] J. Park, J. H. Kang, S. J. Kim, X. Liu, and M. L. Brongersma, Dynamic reflection phase and polarization control in metasurfaces, Nano Lett. 17, 407-413 (2017). doi: 10.1021/acs.nanolett.6b04378.

[32] M. H. Tyboroski, N. R. Anderson, and R. E. Camley, An effective medium study of surface plasmon polaritons in nanostructured gratings using attenuated total reflection, J. Appl. Phys 115, 013104 (2014). doi.org/10.1063/1.4856255

[33] E. Kretschmann, Die Bestimmung optischer Konstanten von Metallen durch Anregung von Oberflächenplasmaschwingungen, Zeitschrift für Phys. 241, 313-324 (1971). doi: 10.1007/BF01395428.

[34] V. Fedotov, Metamaterials, in Springer Handbook of Electronic and Photonic Materials, edited by S. Kasap and P. Capper (Springer, Cham. 2017) p. 1. https://doi.org/10.1007/978-3-319- 
48933-9_56

[35] J. Bai et al., Embedded optical waveguides fabricated in SF10 glass by low-repetition-rate ultrafast laser, Appl. Opt. 52, 7288-7294 (2013). doi: 10.1364/AO.52.007288.

[36] T. Li, Structures, parameters, and transmission properties of optical fibers, Proceedings of the IEEE 68, 1175-1180 (1980). doi: 10.1109/PROC.1980.11825.

[37] C. Yeh, Guided-Wave Modes in Cylindrical Optical Fibers, IEEE Transactions on Education, E-30, 43-51, (1987). doi: 10.1109/TE.1987.5570585.

[38] B. Heinrich, and V. F. Meshcheryakov. Transmission of an Electromagnetic Wave by a Ferromagnetic Metal in the Antiresonance Region. Sov. Phys. JETP 32, 232-237 (1971).

[39] R. E. Camley, Nonreciprocal surface waves, Surf. Sci. Rep. 7, 103-187 (1987). doi: 10.1016/0167-5729(87)90006-9.

[40] R. Macêdo and R. E. Camley, Engineering terahertz surface magnon-polaritons in hyperbolic antiferromagnets, Phys. Rev. B 99, 014437 (2019). doi: 10.1103/PhysRevB.99.014437.

[41] P.G. Baity, D.A. Bozhko, R. Macêdo, W. Smith, R.C. Holland, S. Danilin, V. Seferai, et al., Strong magnon-photon coupling with chip-integrated YIG in the zero-temperature limit, Appl. Phys. Lett. 119 (3), 033502 (2021). https://doi.org/10.1063/5.0054837.

[42] R. Macêdo, R.C. Holland, P.G. Baity, L.J. McLellan, K.L. Livesey, R.L. Stamps, M.P. Weides, D.A. Bozhko, Electromagnetic approach to cavity spintronics, Phys. Rev. Appl. 15, 024065 (2021). https://doi.org/10.1103/PhysRevApplied.15.024065.

[43] L. Remer, B. Lüthi, H. Sauer, R. Geick, and R. E. Camley, Nonreciprocal Optical Reflection of the Uniaxial Antiferromagnet $\mathrm{MnF}_{2} . \quad$ Phys. Rev. Lett. 56, 2752 (1986). https://doi.org/10.1103/PhysRevLett.56.2752 